Medicine Updates Faculty of medicine https://muj.journals.ekb.egdean@med.psu.edu.eg January 2022, volume 8 , issue 8

\title{
" How to Use Quality Tools to Prevent Adverse Drug Reactions in Hospitalls?"
}

\author{
Authors \\ Alshimaa Mohamed Sabry ${ }^{\text {1. }}$; Mohamed Hosny Habashy ${ }^{2}$; Ahmed \\ Mohamed Nageeb ${ }^{3}$; Salma Nasser Nour ${ }^{4}$; Nada Elsayed Elsamahy ${ }^{5}$; Maisara \\ Ibrahim Alameldeen ${ }^{6}$; Saad Ahmed Abdel Aziz ${ }^{7}$; Mohamed Ahmed \\ Elkiky $^{8}$; Dina Mohamed Elsayd ${ }^{9}$

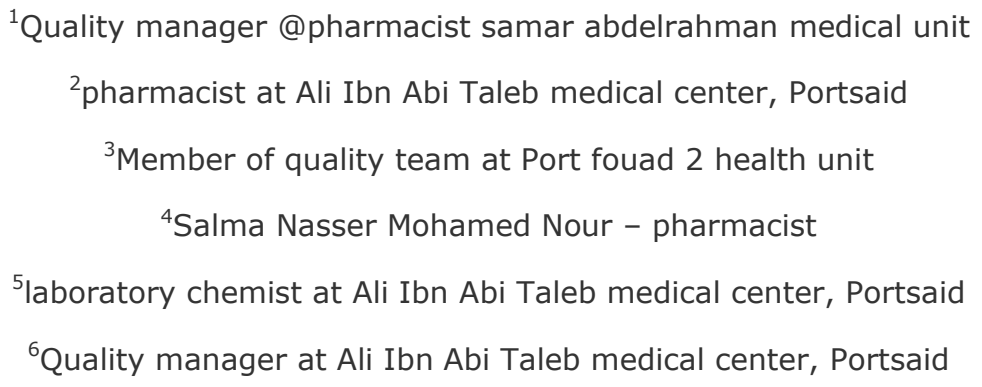




\section{$\underline{\text { Abstract }}$}

Adverse drug reactions (ADRs) and Drug-Drug interactions (DDIs) remain an important clinical problem in modern healthcare systems, especially, with the rising complexity of therapeutics, ageing population and multi-morbidity. There is no doubt that the unpleasant impacts resulting from inaccurate or wrong dose which used in prophylaxis or treatment, will cause significant harm to the patient. Therefore, it is important to shed the light on these such cases in order to avoid further harm consequences. ADRs can be happened due to involvement of T-lymphocyte or IgE-mediated allergy. Hypersensitivity reactions have been highlighted in recent years, so, allergy test is a necessary step before taking medications that may cause hypersensitivity react ion. This article summarizes some of the key facts about ADRs and explores aspects related to their prevention, diagnosis, reporting and management in current clinical practice using strategies, procedures and tools for quality improvement to avoid any probable mistakes. The article will also illustrate the key role of healthcare practitioners in documenting and reporting the ADRs correctly.

\section{$\underline{\text { Key words }}$}

ADRs (Adverse Drug Reactions), WHO (World Health Organization), Quality, Tools, Pareto Chart (a quality tool), Fishbone (a quality tool), NHS (English national health service), NPSA (the National Patient Safety Agency).

\section{$\underline{\text { Introduction }}$}

The impacts that result from administration of medications called "Adverse Drug Reactions (ADRs)". The definition of ADRs according to World Health Organization (WHO) is "any sudden or gradual harm that happen as a response to medication, and happen at ordinary doses used in human beings to avoid a disease, help in diagnosis, treat any illness, or for the modifications of physiological functions. Because of their consequences on people health, ADRs are classified economically as a major problem for healthcare systems. 
Adverse drug reactions (ADRs) are considered a main cause of death and morbidity in both hospitalized and outpatient patients. ADRs are on the list of most ten leading reasons of death in many countries.

As a result, ADRs must be investigated to raise patient awareness of ADRs and encourage health care providers to report ADRs in order to reduce the risk.

ADRs account for about 5\% of all hospital admissions, and about $20 \%$ of inpatient will encounter at least one ADR during their hospitalization. ADRs can be affected by age, gender, genetics, polypharmacy, dose of medication, environmental impacts, and other health factors such as disease conditions. One of the influential causes of morbidity and mortality is ADRs, so, we need to reduce the harmful effects caused by ADRs by right diagnosis, assessment, treatment, and prevention.

Therefore, pharmacovigilance is a major element of effective drug systems, clinical practice and public health programs. World Health Organization defines pharmacovigilance as "the science and activities related to the detection, evaluation, understanding, and prevention of adverse reactions to medications or any other medicine-related problems, especially, long term and short term side effects of medications".

\section{Benefits of pharmacovigilance:}

Pharmaceutical companies' aggressive promotion for new drugs, and the resulting rapid exposure of many numbers of patients to these medications within a fixed period of time, make the development of a project for international assessment of drug safety issues, a necessity. These activities necessitate an effective and efficient pharmacovigilance scheme, to ensure drug safety. For example, Thalidomide tragedy occurred in 1961, forced the WHO to establish a Program called "International Drug Monitoring". Hence, the majority of countries have developed adverse drug reaction (ADR) reporting systems. [1] 
So, the aim of this present study is inspection for adverse drug reactions, adverse drug-drug interactions related to the pharmacotherapy used for treatment of patients in hospitals.

In 2001, Internationally, Egypt decided to join the Drug Monitoring program of world health organization. In addition to this, Egypt implemented Egyptian Pharmaceutical Vigilance Center (EPVC) in December 2009 after eight years of its joining to WHO drug monitoring program.[2]

EPVC helps healthcare systems in gathering the reports submitted by doctors, pharmacists or any other personnel work at the health care facility and patients by the utilization of "yellow cards" and the forms that were designed by the

\section{Council of International Organizations}

for Medical Sciences (CIOMs) which also reported by Marketing Authorization Holders (MAHs).

EPVC website contains a form of "yellow card" and anyone can easily submit it online or download it or even get it from pharmacovigilance coordinator that exists at any health care facility. Reports could be sent by fax, by mail, personally by hand, by telephone or submitted online.

\section{$\underline{\text { Aim of the work }}$}

Highlighting risks of Adverse drug reactions and finding the precautions, procedures and policies to prevent drug related adverse reactions using the quality tools such as (fishbone, flow chart and Pareto chart).

\section{Methodology}

In this qualitative study we will use three quality tools:

\section{- Flowchart}

- fishbone diagram

- Pareto chart 
Let's have some brief notes about these tools.

\section{Flow chart}

It can be named:-( process flowchart), (process flow diagram), (macro flowchart), (top-down flowchart), (detailed flowchart). [3]

A flowchart is a diagram that represents a work flow or process and also can be described as a diagram that represent an algorithm, a step-by-step approach to solve an issue.

The flowchart represents the steps as boxes of different types, and their sequence by connecting the boxes with lines and arrows. This diagrammatic representation gives a solution to a specific problem. Flowcharts are used to analyze, design, document or manage a process or program in different scopes.[4]

\section{Uses of The Flowchart}

- To know how a process is done.

- To illustrate how a process is done.

- To implement a communication method within the team sharing the same process.

- To help quality team to improve any process.

- To plan a project.

- To document a process.

\section{Flowchart Basic Procedure}

- Definition of the process to be diagrammed.

- Choosing the title of the work paper.

- Discuss the outlines of the process \{determining the start and the end step of the process $\}$, Discuss and choose the amount of details in the diagram.

- Brainstorm step for any activity happens.

- Writing each activity on a piece of paper or a card. 
- Arranging the activities in correct way.

- After determining the order of all activities, draw arrows to show the flow of the process.

- Finally, revision of the flowchart with fellows who are involved in the process. Such as, (customers, suppliers or workers) and make sure that all the process personnel agree that the process is made correctly.[5]

\section{FLOW CHART OF DETECTION AND CLASSIFICATION OF ADRS}

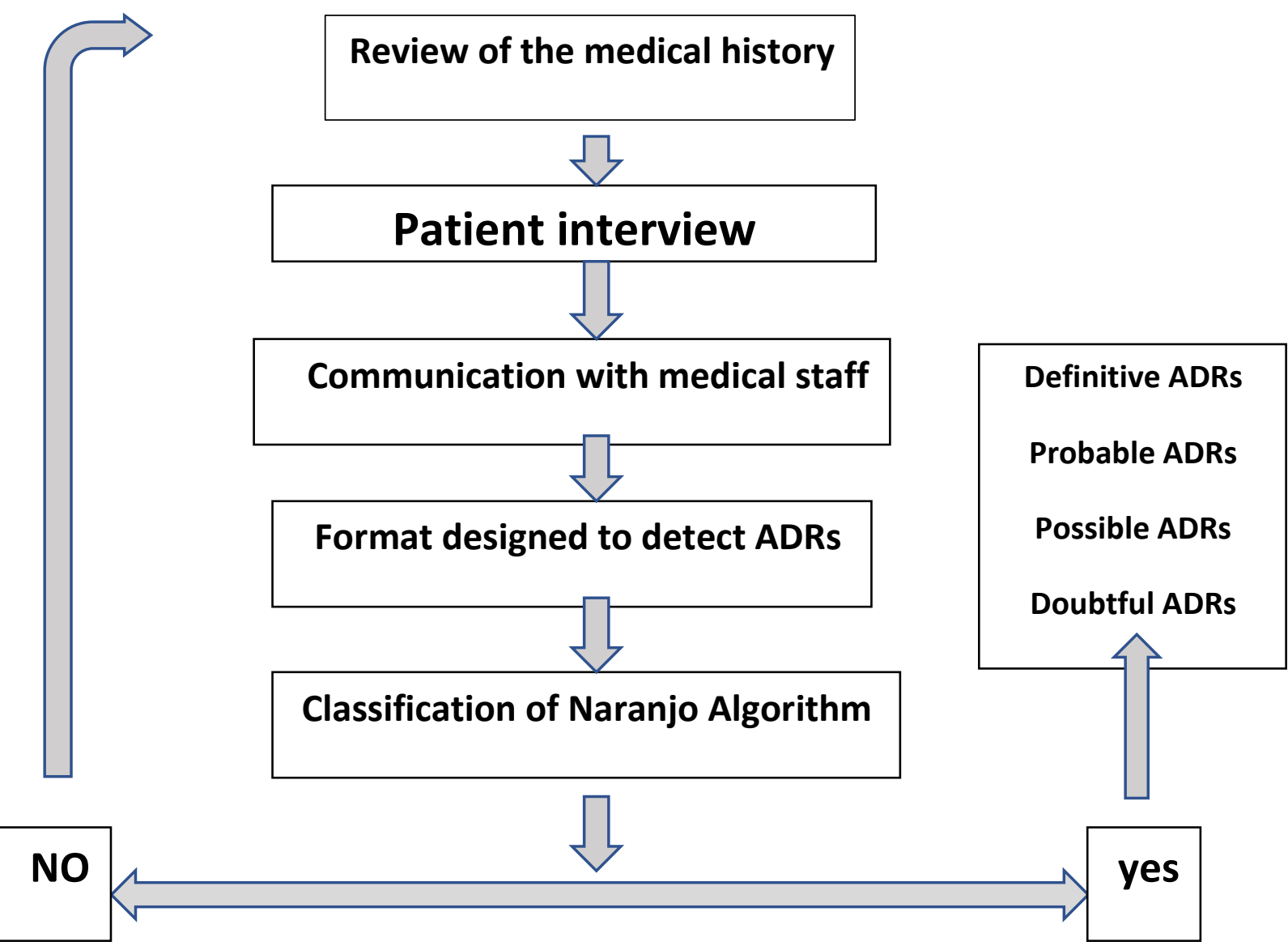

\section{Fishbone diagram}

A fishbone diagram defined as a graphic tool used to present the probable reasons of a dilemma. By this tool we can go deeper to discover the main causes and analyze these reasons. Fishbone diagram techniques are brainstorming then results would be put in specific template. this technique should reach to the causes and effects of any issue or problem. 
Dr. Kaoru Ishikawa, has designed the fishbone diagram to help workers to avoid solutions that only address the symptoms of big problems. Fishbone diagrams are one of seven basic quality tools and are used in the "analyze" which is a phase of Six Sigma's DMAIC (define, measure, analyze, improve, control) approach to problem-solving.

A Fishbone, it is a diagram used as a tool to visualize probable causes of a problem and to categorize these causes so as to identify the Root reasons of the dilemma, in RCA (Root Cause Analysis) Fishbone is Used to detect causes and its effect efficiently, after brainstorming the group for Causes which are possible to affect the problem, after that, the group move to Facilitator to rate it and level its importance and to describe in a diagram, the diagram will seems like a Fish skeleton, mostly, Fish bone diagram include smaller Fishbone diagrams over every large BONE branching from main bone, the smaller Fishbone branches describes details of the cause and its effect on the problem

Japan expert of Quality "Dr. Kaoru Ichikawa "is credited with presenting the fishbone diagram as one of the Basic seven Quality analysis tools in relation to six sigma "DMAIC" approach for problem solving, that's why it may be called Ichikawa Diagram, and in relation it may be called Cause and Effect Diagram AS "DMAIC" is representing the six phases of (Define, measure, analyze, improve, Control)

How to Create a Fishbone,

Following Steps are used during team meeting once identifying the problem to be studied so it may be written on whiteboard or on flipchart

1. Making a list of statements in box representing the problem, that will be the head of fishbone

2. Generic Categories including methods, material, people, equipment and environments or skills, categories are listed and the least -at least- four causes are identified and branched off the spine of the fish 
3. Any supporting info are located down to the corresponding branch cause they mostly will include Questioning methods such as "5 WHYs "and "4 Ps"

4. The Root Cause Should be identified after Breaking Down Causes of Causes, time after time.

\section{The reasons why we use Fishbone diagram:}

To find the potential causes of a problem.

To develop a product that addresses issues within current market offerings.

To eliminate the weak areas in a business process.

To ensure that any corrective plans set by management will resolve the problem.[6]

\section{Example of a fishbone diagram}

The following graphic is an example of a fishbone diagram with the problem "Adverse drug reactions" 


\section{FISHBONE METHOD}

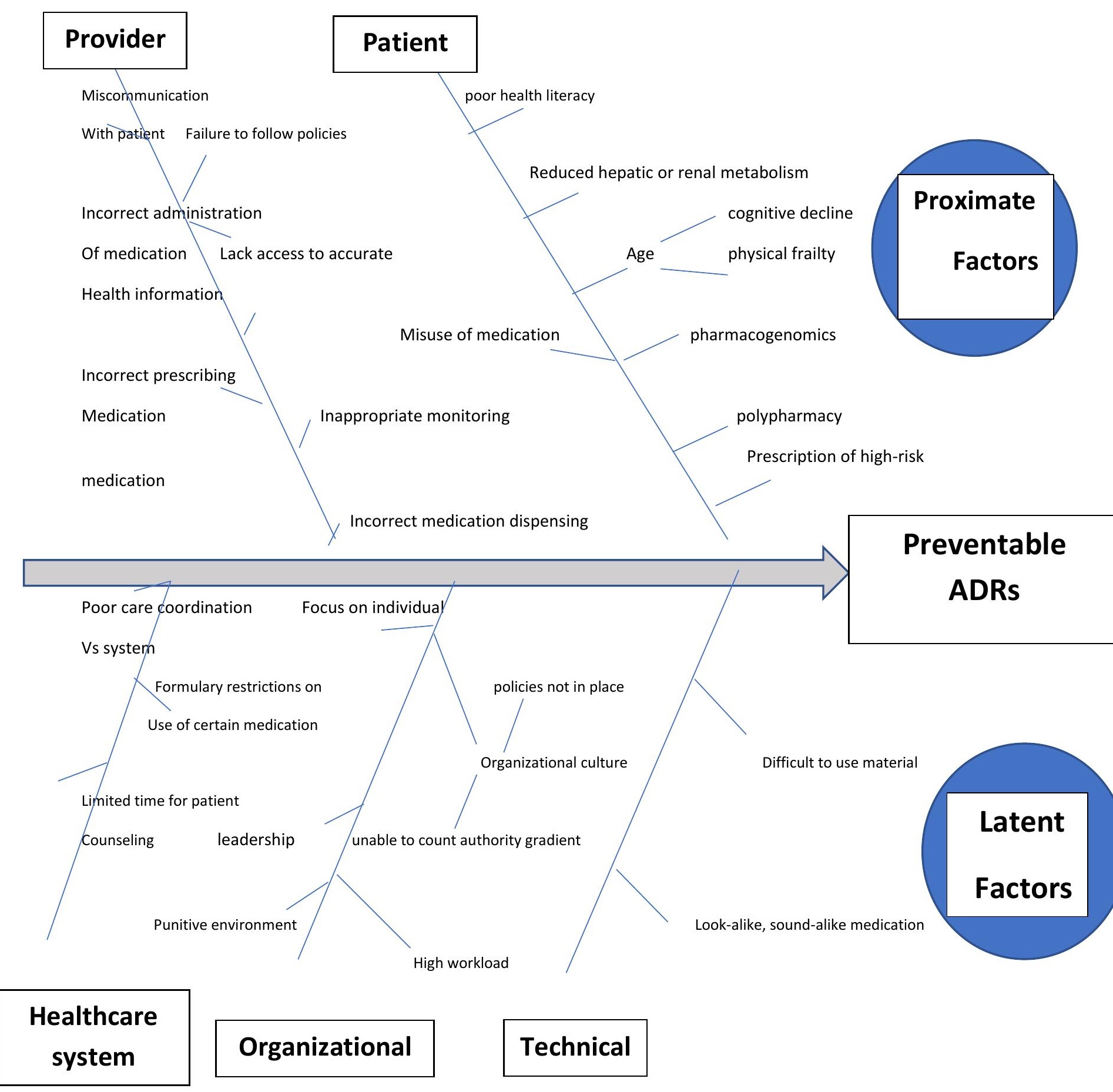

\section{Pareto chart}

A Pareto chart is a type of chart that consists of line graphs and bars, where values are drawn in descending or ascending orders by bars, and the cumulative 
total is represented by the line. The chart is named related to Vilfredo Pareto, an Italian economist.

The vertical axis on the left, represents the frequency of occurrence, but it can represent another significant unit of measuring. The vertical axis on the right, is the cumulative percentage of the total number of occurrences. Because the values are in decreasing order, the cumulative function is a concave function.

The aim of the Pareto chart is to illuminate the top significant factors. In quality control, Pareto charts are useful in setting up priorities in order to observe the overall improvement. It represents the most common causes of defects, or the most frequent causes for patient complaints. Wilkinson (2006) developed an algorithm for making statistically-based limits for each bar in the Pareto chart.

These charts can be generated by simple spreadsheet programs, specialized statistical software tools, and online quality charts generators.

The Pareto chart is one of the seven basic tools of quality control[7]

\section{Uses of The Pareto Chart}

- For analyzing data of problems or causes in a process according to their repeat.

- For prioritizing the causes of any problem or issue.

- For using your data to communicate with others. [8] 
PARETO CHART

\begin{tabular}{|l|l|c|l|}
\hline Cum \% & Percentage & $\begin{array}{c}\text { Number of } \\
\text { occurrence }\end{array}$ & Reasons of adverse drug reactions \\
\hline$\underline{31.25 \%}$ & $\underline{31.25 \%}$ & 50 & Prescribed wrong dose \\
\hline$\underline{\mathbf{5 7 . 5 0 \%}}$ & $\underline{\mathbf{2 6 . 2 5 \%}}$ & 42 & Duplication of the dose \\
\hline$\underline{\mathbf{7 0 . 0 0 \%}}$ & $\underline{12.50 \%}$ & 20 & $\underline{\text { Drug-drug interactions }}$ \\
\hline$\underline{\mathbf{7 9 . 3 8 \%}}$ & $\underline{9.38 \%}$ & 15 & $\underline{\text { Food-drug interactions }}$ \\
\hline$\underline{\mathbf{8 5 . 6 3 \%}}$ & $\underline{6.25 \%}$ & 10 & $\underline{\text { Allergy }}$ \\
\hline$\underline{\mathbf{9 0 . 6 3 \%}}$ & $\underline{\mathbf{5 . 0 0 \%}}$ & $\mathbf{8}$ & $\underline{\text { Body weight and fat distribution }}$ \\
\hline$\underline{\mathbf{9 3 . 7 5 \%}}$ & $\underline{3.13 \%}$ & 5 & Wrong prescribed drug due to wrong diagnosis \\
\hline$\underline{\mathbf{9 6 . 8 8 \%}}$ & $\underline{3.13 \%}$ & 5 & Wrong dispensed drug \\
\hline$\underline{\mathbf{9 8 . 7 5 \%}}$ & $\underline{1.88 \%}$ & 3 & $\underline{\text { Reasons related to the patient(age/gender/pregnancy and }}$ \\
\hline$\underline{100.00 \%}$ & $\underline{1.25 \%}$ & 2 & $\underline{\text { lactation/smoking/alcohol drinker( }}$ \\
\hline- & - & 160 & Patient with renal or hepatic dysfunctions \\
\hline
\end{tabular}

\section{REASONS OF DRUG REACTIONS}
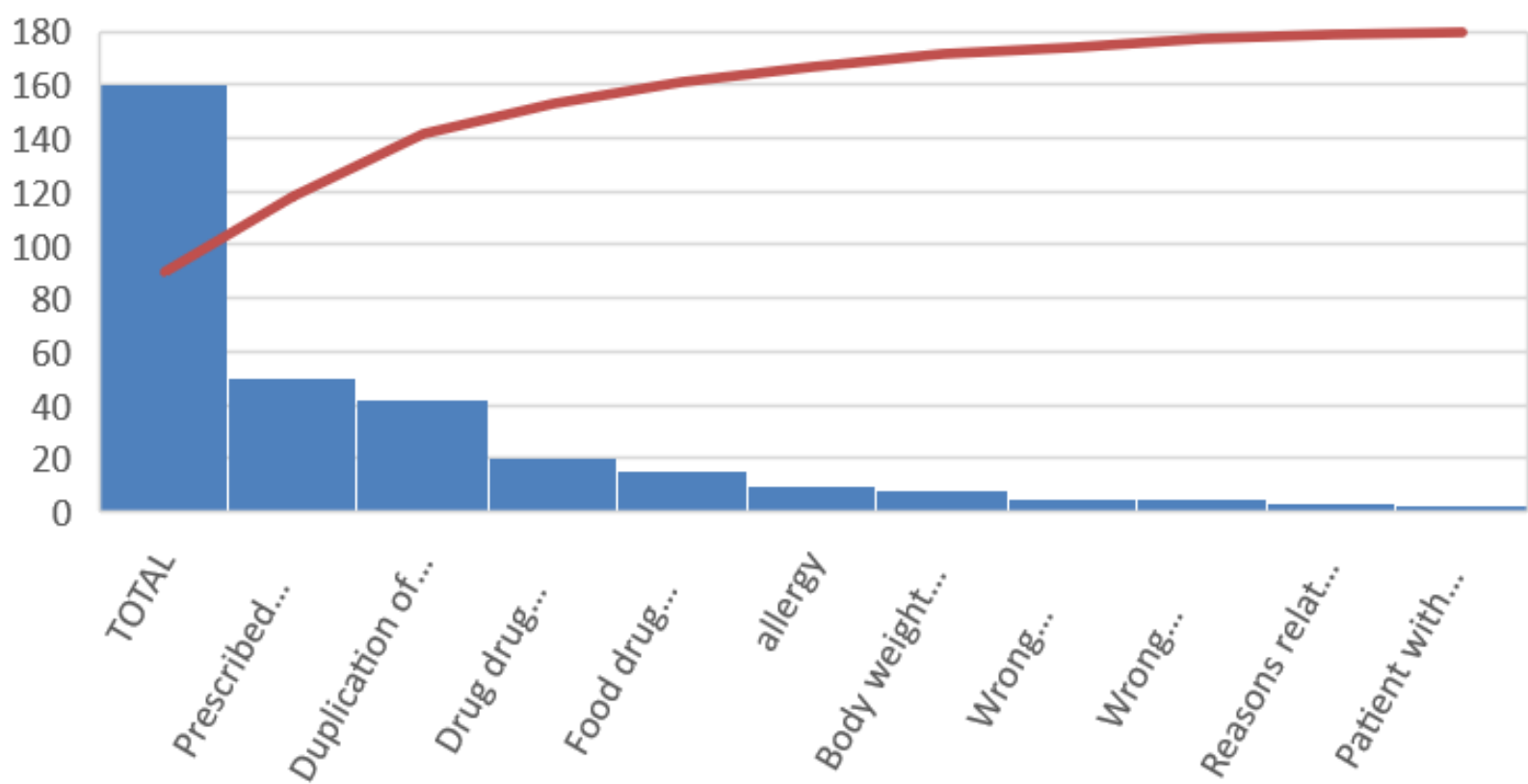


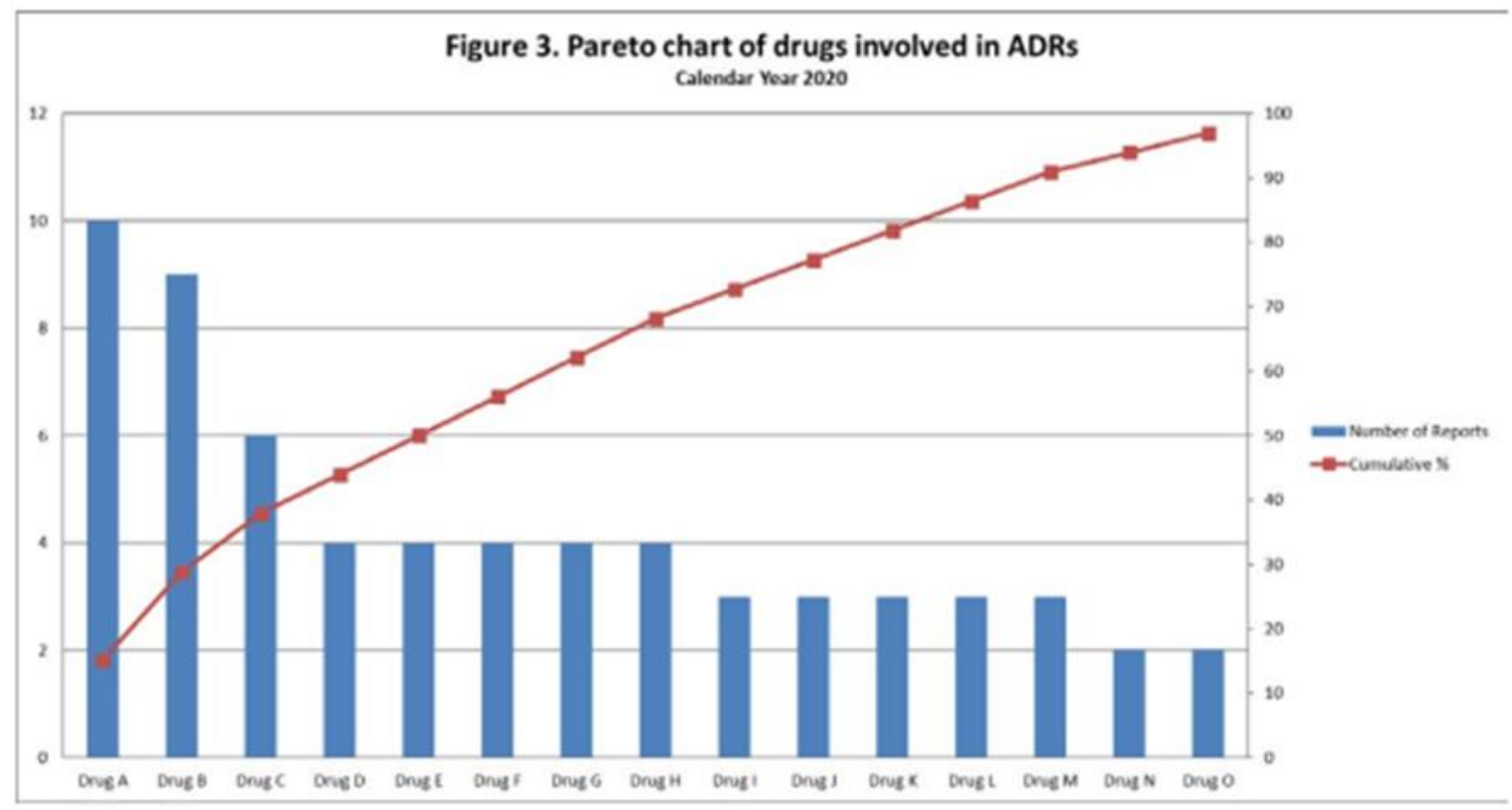

Figure 3. ADR trending by involved drug using Pareto principle. In the example, Drug A was the top reported medication involved in ADR events which can be used as a signal to warrant additional investigation.

\section{$\underline{\text { Discussion }}$}

In this research, we plan to describe the hazardous effects of different ADRs on patient and also finding the main reasons that cause any serious adverse effects and using the quality tools which are (Flow chart, Fish bone and BARETO Chart).

First things first, we find that the most common causes of the ADRs by Fish bone diagram and then we arrange these causes in descending order. There are four causes compose about $80 \%$ of the total causes which are (Prescribed wrong dose, Duplication of the dose, Drug-drug interactions and Food-drug interactions.

On the other hand, in another research there was another main cause for ADRs like in (Philip M. Hopkins, Adverse Drug Reactions Abhinav Kant, in Pharmacology and Physiology for Anesthesia, 2013).[9] 
If the drug is not administered to a patient in right way, Adverse drug reactions happen. In the last two decades, NHS England (and the National Patient Safety Agency NPSA) and the Institute of Medicine in the United States have both compiled massive data showing that this is a significant and widespread issue in healthcare facilities and drug administration mistakes are the most preventable cause of patient harm. Although the majority of these reported errors lead to minimal or no harm, in anesthesia, it might lead to significant impacts. There is a study executed in United Kingdom. More than 12,500 incidents were reported and documented. These incidents were medication errors represent about $9 \%$ of total cases. Only $(1.3 \%)$ was due to huge harm or mortality. By analysis of reports to the NPSA about drug errors in ICU illuminate that about $\mathbf{2 4 0 0}$ incidents documented, where about 350 different medications contributed in these errors, with Gentamicin, Norepinephrine and also Morphine were occupy the most reasons for errors in this study. The definition of anesthetic drug error is any mistake that occur frequently every 133 anesthetic cases. These mistakes could be prevented by using Double-check technique for drugs to prevent administration mistakes.[10]

On the other side, for specific medications, insufficient information about dosing among doctors could cause false dose. Drug development in a non-anesthetic context applies to some medications which were delivered to clinical practice at a time when making legislation was not potent. For incidence, Morphine has a conventional dose for strong pain after operations, which is $\mathbf{0 . 1 5} \mathbf{~ m g} / \mathbf{k g}$ given IM. This dose represents the effective dose for battlefield casualties. However, one can now accept that battlefield casualties are an unsuitable pattern for postoperative pain because the pain tolerance of those wounded in fighting is elevated due to the neuro-humoral response to the condition.

About 179,000 Americans died from ADRs and over one million were injured as a cause of ADRs in 2008 in a recent meta-analysis of prospective ADR studies 
which showed that Delayed-Time Related is the main cause for ADRs in USA.[10]

Some ADRs are not identified until a sufficient amount of time has passed after the first administration of the drug. Nitrous oxide has been the ability to raise the risk of Megaloblastic bone marrow changes and subsequent neurologic complications (subacute combined degeneration of the spinal cord) due to vitamin B12 deficiency. Short exposure for few hours, can lead to bone marrow changes and more expanded exposure for many days can lead to Agranulocytosis. Vitamin B12 has cobalt ion which could be oxidized by nitrous oxide NO, so will prevent it from activating methionine synthase and stopping the conversion of homocysteine into methionine, thymidine, and tetrahydrofolate is the responsibility of this enzyme.[10]

\section{$\underline{\text { Conclusion }}$}

From the results of this review article the following are concluded:

1- Pharmacists play an important role to help the implementation of an ADR program.

2- Programs should pay attention to surveillance, total documentation in Medical files, and education to achieve an overall objective of minimizing the risk and severity of ADRs in health care sector.

3- Assessment, finding, documenting and education of patients and helth care professionals on ADRs should be done by all pharmacists

4- Training of physicians is a necessary task for any hospital because most of reported ADRs were because of writing wrong dose in prescription.

5- Patient education about the nature and properties of his medications contributes in reducing of wrong dose related ADRs. 


\section{6- Recommendations}

From the results of this research the following are recommended:

Pharmacists connect between health care system and customers who are the patients themselves before and during the course of medication regimen. Pharmacists must be qualified to give precious information about medications, and their side effects as well as drug-drug interactions. This would help mostly in monitoring and controlling side effects, and help in implementing and constructing an improved system in health care facilities.

1- Better approaches should be devised for reporting and assessing ADR.

2- For correct diagnosis and managing ADR medical professionals should be trained.

3- Pharmaceutical companies should strive to reduce the adverse effect of a drug.

4-Further research should be conducted in the area of ADRs.

5-we should implement the quality tools in the hospital's pharmacy to avoid the ADRs.

7- We should use definite policies and procedures to avoid ADRs.

8- We should raise patient awareness towards the harmful results of ADRs is an essential issue.

\section{Summary}

Adverse drug reactions (ADRs) are a main cause of death and morbidity in both hospitalized and outpatient patients as it causes modifications of physiological function in the body leading to harmful consequences. Pharmacovigilance is a key component of effective drug regulation systems which monitors and assess the drug usage, and discovers and documents any ADRs occur. Prescribed wrong dose, duplication of the dose, drug-drug interactions and allergy represent about $\mathbf{8 0 \%}$ of the causes of ADRs according to our findings after doing BARETO

\section{Chart.}


Improving patient care and maintaining the safe drug administration are the key targets for any healthcare quality system. Therefore, the process of education of both doctors and patients about medications effects and raising the awareness level about ADRs reduce the risk and complications of ADRs.

Full documentation in medical files, reporting to and Oreviewing of ADRs by a responsible committee gives a chance for continuous prevention of any possible adverse drug reactions. Qualified Pharmacists play significant function in monitoring side effects as they participate into designing and implementing a strong perfect system related to ADRs in their healthcare systems.

Finally, Healthcare systems must pay more attention to ADRs reporting as well as setting up policies and procedures to diminish all potential adverse drug reactions.

\section{$\underline{\text { References }}$}

1- Reference[1]:

www.researchgate.net/publication/335836807_Adverse_drug_reactions_ of_antineoplastic_and_immunomodulating_agents_reported_to_the_Egy ptian_Pharmaceutical_Vigilance_Center

2- Reference [2]: https://ejmr.journals.ekb.eg/article_175723_21510_html

3- Reference [3]: https://asq.org/quality-resources/flowchart

4- Reference [4]: SEVOCAB: Software Systems Engineering Vocabulary. Term: Flow chart. Retrieved 31 July 2008.

5- Reference [5]: https://asq.org/quality-resources/flowchart

6- Reference [6]: https://whatis.techtarget.com/definition/fishbone-diagram

7- Reference [7]: Nancy R. Tague (2004). "Seven Basic Quality Tools". The Quality Toolbox. Milwaukee, Wisconsin: American Society for Quality. p. 15. Retrieved 2010-02-05.^ "What is a Pareto Chart? Analysis \& Diagram | ASQ". asq.org. Retrieved 2019-05-10.

8- Reference [8]: https://asq.org/quality-resources/flowchart 
9- Reference [9]: Adverse Drug Reactions Abhinav Kant, Philip M. Hopkins, in Pharmacology and Physiology for Anesthesia, 2013).

10- Reference[10]:https://journals.plos.org/plosone/article?id=10.1371/journa 1.pone. 0004439 